Virginia Commonwealth University vCU Scholars Compass

2012

\title{
Tuning magnetic properties of graphene nanoribbons with topological line defects: From antiferromagnetic to ferromagnetic
}

Min Kan

Peking University

Jian Zhou

Peking University

Qiang Sun

Virginia Commonwealth University

See next page for additional authors

Follow this and additional works at: http://scholarscompass.vcu.edu/phys_pubs

Part of the Physics Commons

Kan, M., Zhou, J., Sun, Q. et al. Tuning magnetic properties of graphene nanoribbons with topological line defects: From antiferromagnetic to ferromagnetic. Physical Review B, 85, 155450 (2012). Copyright (C 2012 American Physical Society.

\section{Downloaded from}

http://scholarscompass.vcu.edu/phys_pubs/55

This Article is brought to you for free and open access by the Dept. of Physics at VCU Scholars Compass. It has been accepted for inclusion in Physics Publications by an authorized administrator of VCU Scholars Compass. For more information, please contact libcompass@vcu.edu. 
Authors

Min Kan, Jian Zhou, Qiang Sun, Qiang Wang, Yoshiyuki Kawazoe, and Puru Jena 


\title{
Tuning magnetic properties of graphene nanoribbons with topological line defects: From antiferromagnetic to ferromagnetic
}

\author{
Min Kan, ${ }^{1, *}$ Jian Zhou, ${ }^{1, *}$ Qiang Sun, ${ }^{1,2,3, \dagger}$ Qian Wang, ${ }^{2,3}$ Yoshiyuki Kawazoe, ${ }^{4}$ and Puru Jena ${ }^{3}$ \\ ${ }^{1}$ Department of Materials Science and Engineering, College of Engineering, Peking University, Beijing 100871, China \\ ${ }^{2}$ Center for Applied Physics and Technology, Peking University, Beijing 100871, China \\ ${ }^{3}$ Department of Physics, Virginia Commonwealth University, Richmond, Virginia 23284, USA \\ ${ }^{4}$ Institute for Material Research, Tohoku University, Sendai, 980-8577, Japan
}

(Received 22 October 2011; revised manuscript received 10 January 2012; published 25 April 2012)

\begin{abstract}
Zigzag-edged graphene nanoribbons are antiferromagnetic in cross-edge coupling and unsuitable for spintronics applications. Two new strategies of tuning antiferromagnetism (AFM) to ferromagnetism (FM) in graphene nanoribbons are introduced through topological line defects composed of pentagonal and octagonal rings, and their ability to induce magnetic transition is probed by using density functional theory. The resulting exchange energy is found to be large enough for ferromagnetism to be observed at room temperature. Both strategies are experimentally feasible, and the results suggest that defect engineering may provide a novel path to manipulate the magnetic properties of graphene nanoribbons.
\end{abstract}

DOI: 10.1103/PhysRevB.85.155450

PACS number(s): 31.15.E-, 36.40.Cg

\section{INTRODUCTION}

Graphene, ${ }^{1}$ a two-dimensional (2D) single atomic layer composed of $s p^{2}$ hybridized carbon atoms, and its related structures have attracted considerable research interest due to their novel physical properties. ${ }^{2}$ One of the interesting properties is its intrinsic magnetism related to $p$ electrons, which is advantageous over the conventional $d$ - or $f$-electron based magnetic systems due to weak spin-orbit interaction and long electron spin coherence time. Although the pristine 2D infinite graphene sheet is nonmagnetic (NM), zigzag-edged graphene nanoribbons (ZGNRs) have magnetic edges, which have been theoretically predicted and experimentally confirmed. ${ }^{3-6}$ Although the edge $\mathrm{C}$ atoms in ZGNR couple ferromagnetically within the edge, ${ }^{7}$ they couple antiferromagnetically across the edges, ${ }^{6}$ resulting in no net magnetic moment in the system. This is consistent with Lieb's theorem ${ }^{8}$ of bipartite system derived from the tight binding Hubbard model. The term bipartite means that the system lattices can be divided into two sublattices $\alpha$ and $\beta$, where the hopping integral $t_{x y}=0$ if $x \in \alpha, y \in \alpha$, or $x \in \beta, y \in \beta$. Lieb's theorem states that if the Hubbard Coulomb energy $U$ is positive and constant, the total magnetic moment can be calculated to be $m=\left|n_{\alpha}-n_{\beta}\right| / 2$, where $n_{\alpha(\beta)}$ is the total number of $\alpha(\beta)$ sublattice. In the ZGNRs, $n_{\alpha}=n_{\beta}$, and hence $m=0$. In fact, $\mathrm{C}$ atoms in bipartite lattice favor to couple antiferromagnetically or ferrimagnetically with their nearestneighbor $\mathrm{C}$ atoms. This has been called the antipattern rule. ${ }^{9}$

The total magnetic moment of $0 \mu_{\mathrm{B}}$ hinders further applications of ZGNRs in spintronics. Therefore it is highly desirable to modulate the magnetic coupling between the two edges of ZGNRs from antiferromagnetism (AFM) to ferromagnetism (FM). Currently, a few possible methods have been proposed. ${ }^{10-16}$ For example, Sawada et al. found that injecting carriers (electrons or holes) to ZGNR can yield ferromagnetic coupling between the two edges. ${ }^{10}$ Transition to FM can also be induced by the substrate magnetic field when ZGNRs are deposited on graphene/Ni(111) surface. ${ }^{11}$ However, the energy difference between FM and AFM states is only about $\sim 4 \mathrm{meV}$. Wang et al. ${ }^{12}$ proposed that two-sided oxidation of ZGNRs may be an effective way to obtain FM ZGNRs with higher exchange energy. However, according to the present oxidized graphene patterns, ${ }^{13}$ this is hard to achieve experimentally and may not be stable. ${ }^{14} \mathrm{Xu}$ et al. demonstrated that the transition to FM can be possible when one edge is saturated by two $\mathrm{H}$ atoms and the other edge by one $\mathrm{H},{ }^{15}$ but such precise control is also experimentally not easy. Lin $e t$ al. showed that the ZGNRs with topological line defect composed of two pentagonal and one octagonal rings in one unit cell (which is denoted as 558-defect ZGNRs in the subsequent paragraphs) can be tuned from AFM to FM when their widths are large enough, but the exchange energies are still small. ${ }^{16}$

In this paper we focus on ZGNRs with 558-type line defect where pentagons are connected with octagons, which have been fabricated in experiment through adsorbing two graphene sheets on a $\mathrm{Ni}(111)$ surface with respect to fcc (face-centered cubic) and hcp (hexagonal close-packed) patterns. ${ }^{17}$ This kind of defect is predicted to lead to quasi-one-dimensional (1D) metallic conductivity and has received much attention. ${ }^{16,18-21}$ Using first-principles calculations, we show that the coupling of the two edges in ZGNR with 558-defect is still AFM in its free state, which can be tuned to FM by (1) applying uniaxial strain along the periodic direction or (2) substitutional doping with $\mathrm{B}, \mathrm{N}, \mathrm{Al}$, or $\mathrm{P}$. Both strategies can induce magnetism in the $\mathrm{C}$ atoms associated with the defect line. For nanoribbons with about $2 \mathrm{~nm}$ in width, the FM state can be energetically lower than the AFM state by $10 \sim 50 \mathrm{meV}$ per unit cell, which is large enough to be observed and applied in future experiments. According to Mermin-Wagner theorem, ${ }^{22} \mathrm{O}$. V. Yazyev et $a l^{7,23}$ has stated that the spin-spin correlation length of ferromagnetically coupled ZGNR edge is about $1 \mathrm{~nm}$ under room temperature. Hence, the length of spintronic devices should be within a few nanometers. Nevertheless, we noticed a recent experimental work, ${ }^{24}$ which reported the first observation of energy splitting around the Fermi level of chiral GNRs in the $d I / d V$ spectra. This is an evidence of GNR magnetism suggesting that the GNRs can be used as potential spintronic devices. Some ferromagnetic graphenebased materials have been observed. ${ }^{25}$ Here, we study ZGNRs 
with a topological defect line, which will be helpful to study realistic chiral GNRs with defect lines. And also, if the width of the nanoribbon can be controlled within a few nanometers, the ferromagnetic coupling between the two edges can be retained. We also investigated the width dependence of the exchange energy and showed that larger magnetic coupling can be achieved for narrower 558-defected ZGNRs.

\section{COMPUTATIONAL METHODS}

Our first-principles calculations are based on spin-polarized density functional theory (DFT) using generalized gradient approximation $(\mathrm{GGA})^{26}$ for exchange-correlation potential given by Perdew-Burke-Ernzerhof (PBE). All the calculations are performed using a DMol3 package, ${ }^{27,28}$ and the periodic boundary condition is used to simulate 1D ZGNRs. Dangling bonds on zigzag edges are terminated by hydrogen atoms in order to retain the $s p^{2}$ hybridization of $\mathrm{C}$ atoms. We use a vacuum space of $15 \AA$ in the nonperiodic directions in order to avoid interactions between two neighboring images. The reciprocal space is represented by Monkhorst-Pack special $k$-point scheme ${ }^{29}$ with $9 \times 1 \times 1$ grid meshes. We have used effective core potential with double-numerical plus polarization (DNP) basis set, which includes a $p$ electron polarization function on hydrogen atoms. All the structures are relaxed without any symmetric constraints. The criteria of convergence of energy, force, and displacement are set to be $1 \times 10^{-5} \mathrm{Ha}, 0.002 \mathrm{Ha} / \AA$, and $0.005 \AA$, respectively. To investigate the magnetic coupling between two edges of the 558-defect ZGNRs, we calculate and compared the energies corresponding to FM and AFM, as well as the NM state. The magnetic moments of atoms are analyzed through the Mulliken scheme. The accuracy of our calculation procedure has been tested in our previous work. ${ }^{30}$

\section{RESULTS}

\section{A. Geometric, electronic, and magnetic properties of 558-defect ZGNRs}

We first examine the geometric, electronic, and magnetic properties of ZGNR containing 558-defect line (Fig. 1), where there are nine chains, denoted as 558-defect-9-ZGNR, and the width is about $2 \mathrm{~nm}$. After relaxation, the 558-defect-9-ZGNR retains its planar structure. The $\mathrm{C}-\mathrm{C}$ bond lengths of pentagonal

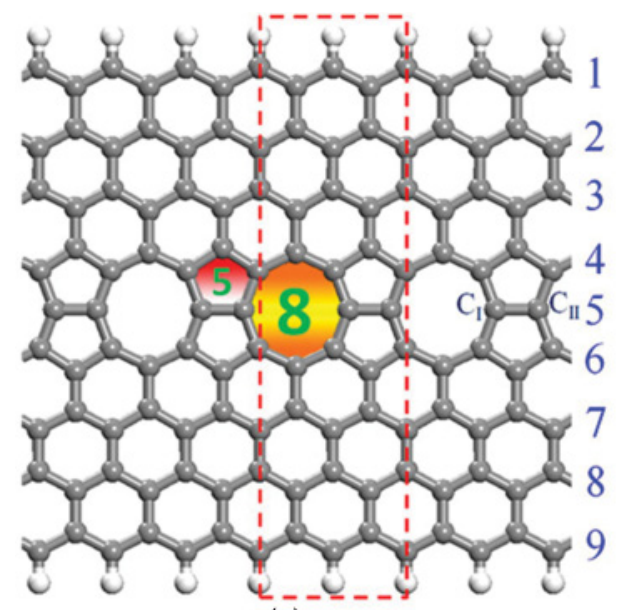

(a)

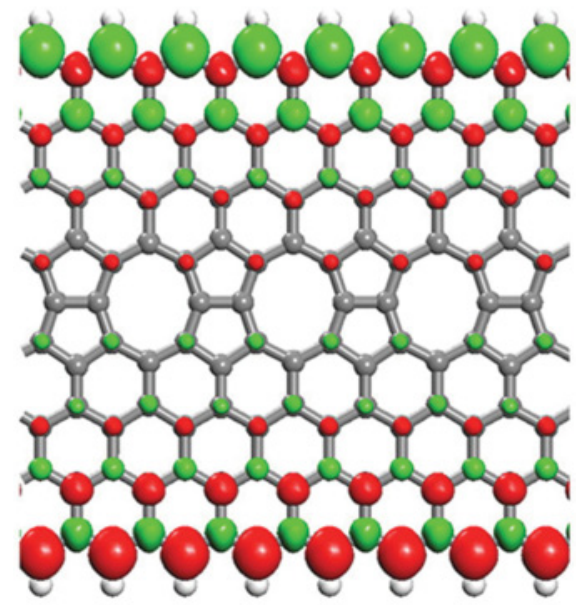

(c)

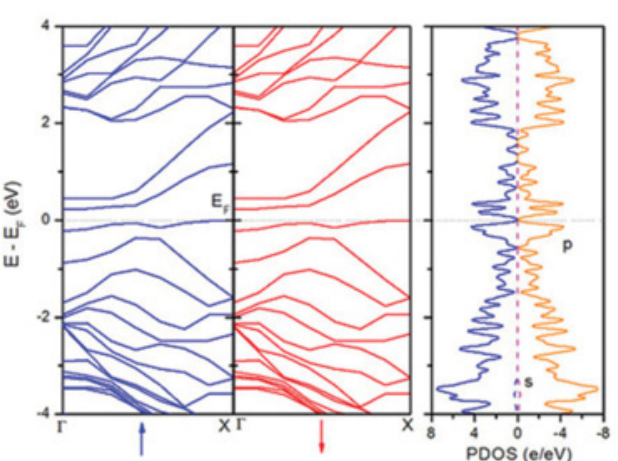

(b)

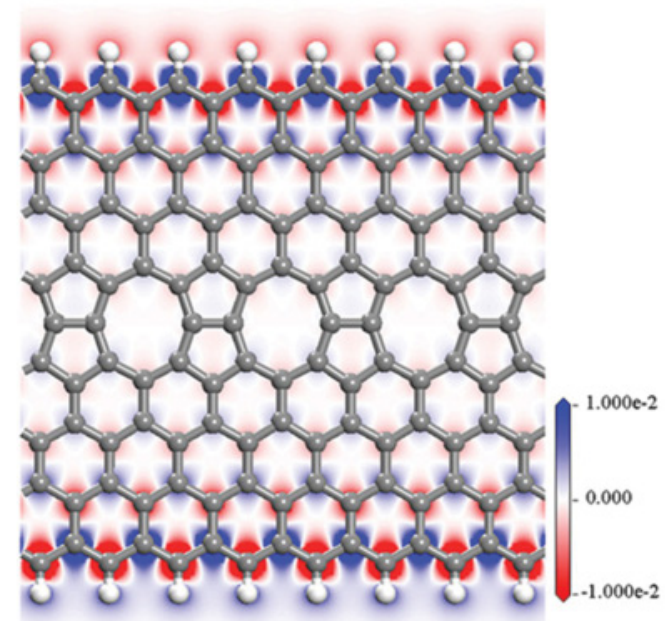

FIG. 1. (Color online) (a) Geometric structure, (b) band structure and PDOS, and (c) iso-surface (left panel with value of 0.01 electron $/ \AA^{3}$ ) and 2D slice (right panel) of spin density of 558-defect-9-ZGNR. The dashed rectangle indicates the unit cell. In the iso-surface plot, green (gray) and red (dark gray) represent positive and negative values, respectively. 
and octagonal rings are within $1.43 \sim 1.45 \AA$, indicating delocalized $\pi$ bonding. The AFM and FM coupling states of the two edges are also calculated, and the AFM state is energetically more favorable than the FM state by $14 \mathrm{meV} / \mathrm{unit}$ cell, consistent with previous report. ${ }^{16}$ For the AFM ground state, contrary to the 2D metallic conductivity, the band structure and partial density of states (PDOS) suggest that the system is a semiconductor with an indirect band gap of $0.22 \mathrm{eV}$. The valence band maximum (VBM) is located at the $\mathrm{X}$ point of the reciprocal space and is contributed by the two edges (not shown here), while the conductance band minimum (CBM) is located at $\Gamma$ points and is contributed by the central defect line. In order to clarify the stability of the 558-defect-9-ZGNR, we investigate the binding energy and compare it with that of 9-ZGNR. Here, the binding energy is defined as $E_{\mathrm{b}}=$ $\left(E_{558}-n_{\mathrm{C}} E_{\mathrm{C}}-n_{\mathrm{H}} E_{\mathrm{H}}\right) / n_{\mathrm{C}}$, where $E_{558}, E_{\mathrm{C}}$, and $E_{\mathrm{H}}$ are the total energy of the investigated system, a $\mathrm{C}$ atom and a $\mathrm{H}$ atom, respectively; $n_{\mathrm{C}}$ and $n_{\mathrm{H}}$ are the number of $\mathrm{C}$ atoms and $\mathrm{H}$ atoms, respectively. The calculated binding energy of 558-defect-9-ZGNR is $-8.13 \mathrm{eV} /$ atom, which is comparable with the perfect pristine $9-\mathrm{ZGNR}(-8.18 \mathrm{eV} /$ atom).

We should point out that the AFM ground state of the 558-defect-9-ZGNR cannot be understood directly through the antipattern rule because the system is not a bipartite lattice. Detailed spin density analysis [Fig. 1(c)] shows that the central $\mathrm{C}$ atoms $\left(\mathrm{C}_{\mathrm{I}}\right.$ and $\left.\mathrm{C}_{\mathrm{II}}\right)$ carry no magnetic moment, and the spins of the $\mathrm{C}$ atoms linked with them are antiparallel.
In the following we suggest that if we can introduce net magnetic moment in the central $\mathrm{C}_{\mathrm{I}}$ and $\mathrm{C}_{\mathrm{II}}$, then the linked $\mathrm{C}$ atoms would carry spins along the same direction, and subsequently the two edges of 558-defect-9-ZGNR will be coupled in the FM configuration.

\section{B. Strategy I: Uniaxial tension}

A well-known classical example of strain-induced magnetism is bulk vanadium, which becomes magnetic when its lattice constant is expanded by $3 \% .^{31}$ Recently, Kuo et al. found that tensile strain along the zigzag direction can greatly enhance local magnetic moments and ferromagnetic stability of a 2D graphene with 558 topological line defects. ${ }^{19}$ Therefore, we can imagine that when uniaxial tension is applied to the 1D 558-defect-9-ZGNR [Fig. 2(a)], the $C_{I}$ and $\mathrm{C}_{\mathrm{II}}$ may become magnetic and AFM to FM transition may also occur. Our detailed calculations confirm this expectation.

In Fig. 2(b) we show the exchange energy $\Delta E\left(=E_{\mathrm{AFM}}-\right.$ $\left.E_{\mathrm{FM}}\right)$ as a function of the lattice constant $c$. Although in the equilibrium state ( $c=4.90 \AA$ ), the AFM state is the ground state. As the lattice gets expanded, the stability of FM state is gradually enhanced. The critical value for $c$ is $5.03 \AA(2.7 \%$ expansion) at which the FM and AFM states are energetically degenerate. Beyond this value the FM state becomes rapidly more stable. For example, for $c=5.15 \AA$ (5\% expansion) $\Delta E$ reaches to $40 \mathrm{meV}$ per unit cell. We find that correspondingly

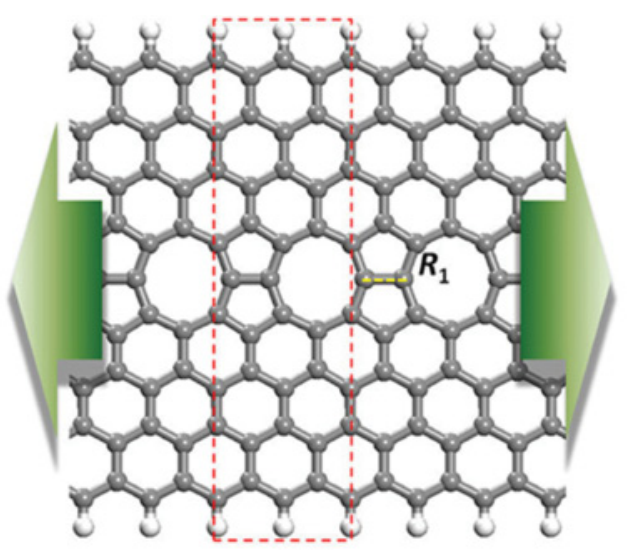

(a)

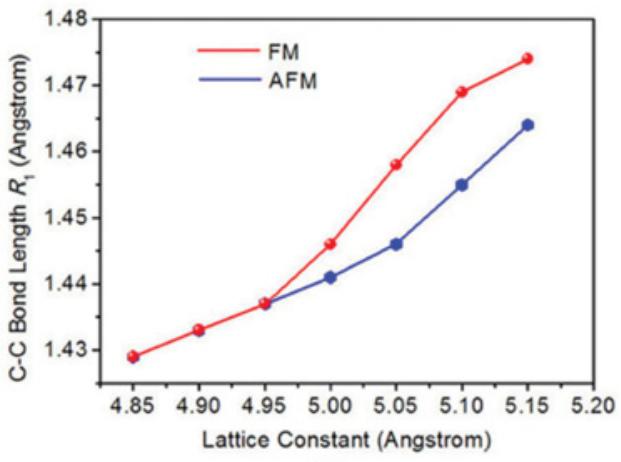

(c)

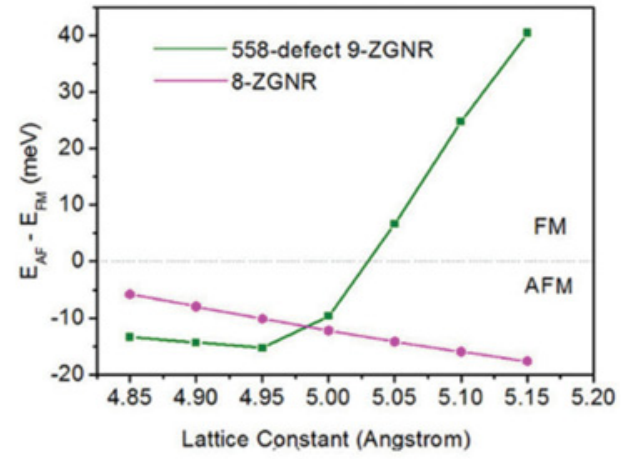

(b)

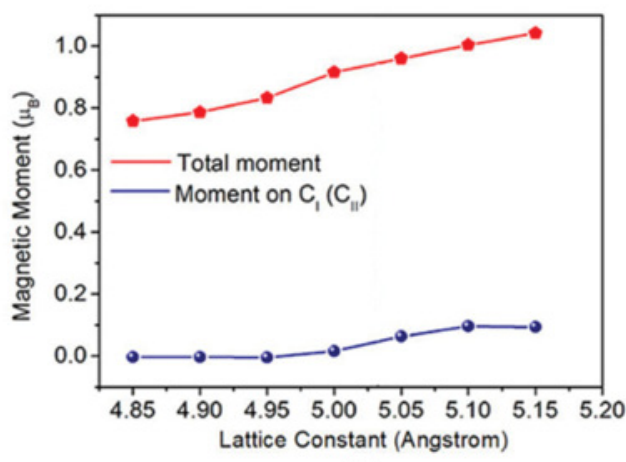

(d)

FIG. 2. (Color online) (a) Illustration of uniaxial tension of 558-defect-9-ZGNR. Variation of (b) exchange energy, (c) $\mathrm{C}_{\mathrm{I}}-\mathrm{C}_{\mathrm{II}}$ bond length $\mathrm{R}_{1}$, and (d) total magnetic moments per unit cell and on $\mathrm{C}_{\mathrm{I}}\left(\mathrm{C}_{\mathrm{II}}\right)$ atom in the FM state as a function of the lattice constant. 

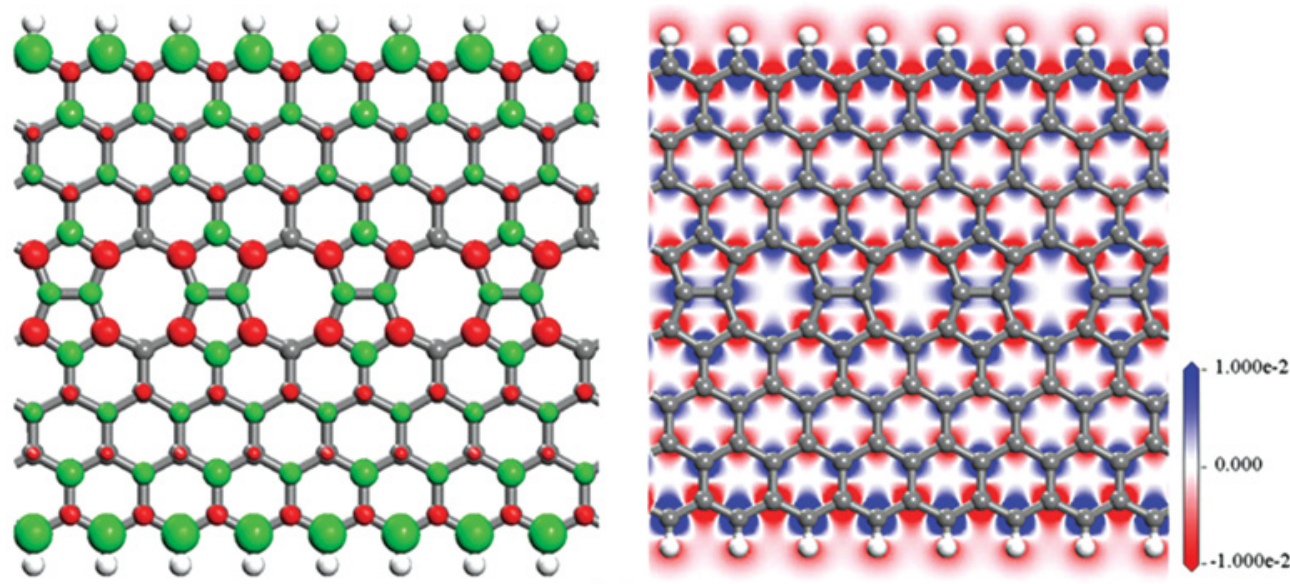

(a)
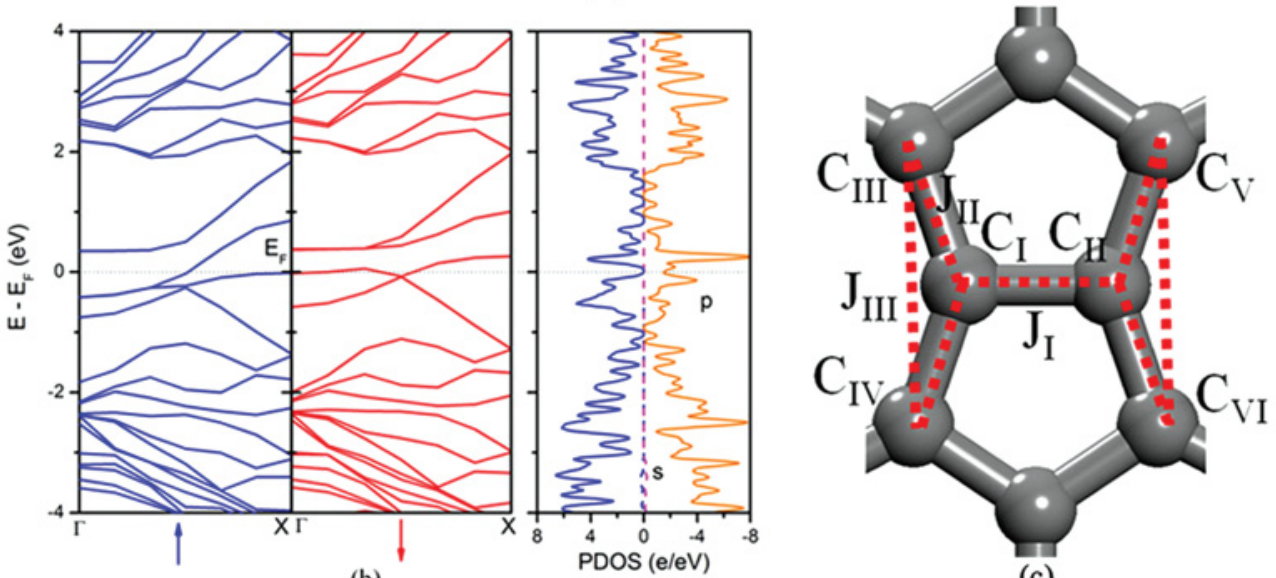

(b)

(c)

FIG. 3. (Color online) (a) Spin density and (b) band structure and PDOS of 558-defect-9-ZGNR at lattice constant $c=5.15 \AA$; (c) magnetic coupling of the topological defect.

the $\mathrm{C}_{\mathrm{I}}-\mathrm{C}_{\mathrm{II}}$ bond length in the FM state is larger than that of the AFM configuration, as shown in Fig. 2(c), indicating weaker bond. Such a weak $\mathrm{C}_{\mathrm{I}}-\mathrm{C}_{\mathrm{II}}$ bond can induce magnetism on the two atoms, which originally were nonmagnetic. In Fig. 2(d) we plot the magnetic moment carried by the $\mathrm{C}_{\mathrm{I}}$ (or $\mathrm{C}_{\mathrm{II}}$ ) atom as a function of uniaxial tension. The data are collected from the 558-defect-9-ZGNR in the FM edge-coupling state. It is observed that the $\mathrm{C}_{\mathrm{I}}\left(\mathrm{C}_{\mathrm{II}}\right)$ atoms are nearly $\mathrm{NM}$ under small tension, while they become magnetic when $c \geqslant 5.00 \AA$. This is consistent with the FM stability and $\mathrm{C}_{\mathrm{I}}-\mathrm{C}_{\mathrm{II}}$ bond weakening. The spin distribution [Fig. 3(a)] for $c=5.15 \AA$ (5\% expansion) clearly shows the FM coupling between the $\mathrm{C}_{\mathrm{I}}$ and $\mathrm{C}_{\mathrm{II}}$ atoms, suggesting that ferromagnetism appears before the complete breakage of $\mathrm{C}_{\mathrm{I}}-\mathrm{C}_{\mathrm{II}}$ bond. From the spin distribution plot, we observe that the $\mathrm{C}$ atoms linked with $\mathrm{C}_{\mathrm{I}}$ and $\mathrm{C}_{\mathrm{II}}$ atoms are all polarized with spin down, in contrast with those in Fig. 1(c). This parallel spin polarization thus induces FM coupled edges in the 558-defect-9-ZGNR. We also calculate the total magnetic moment of the 558-defect-9-ZGNR in the FM edge-coupling configuration [Fig. 2(d)], which clearly shows nonzero value and gradual increment with respect to the lattice constant.

We analyze the magnetic distribution near the topological defect through the Ising model to understand the magnetic coupling transition. As shown in Fig. 3(c), we are interested in the magnetic coupling between the $\mathrm{C}_{\mathrm{I}}$ and $\mathrm{C}_{\mathrm{II}}$, the $\mathrm{C}_{\mathrm{I}}$ and $\mathrm{C}_{\mathrm{III}}$ $\left(\mathrm{C}_{\mathrm{I}}\right.$ and $\left.\mathrm{C}_{\mathrm{IV}}\right)$, the $\mathrm{C}_{\mathrm{II}}$ and $\mathrm{C}_{\mathrm{V}}\left(\mathrm{C}_{\mathrm{II}}\right.$ and $\left.\mathrm{C}_{\mathrm{VI}}\right)$, and the $\mathrm{C}_{\mathrm{III}}$ and $\mathrm{C}_{\mathrm{IV}}\left(\mathrm{C}_{\mathrm{V}}\right.$ and $\left.\mathrm{C}_{\mathrm{VI}}\right)$ atoms. We denote the exchange parameters between them as $J_{\mathrm{I}}, J_{\mathrm{II}}$, and $J_{\mathrm{III}}$, respectively, which are all positive due to the antipattern rule: they favor to couple ferrimagnetically with each other. The total exchange energy can be written as $E=J_{\mathrm{I}} m_{\mathrm{I}} m_{\mathrm{II}}+J_{\mathrm{II}} m_{\mathrm{I}} m_{\mathrm{III}}+J_{\mathrm{II}} m_{\mathrm{I}} m_{\mathrm{IV}}+$ $J_{\mathrm{III}} m_{\mathrm{III}} m_{\mathrm{IV}}+J_{\mathrm{II}} m_{\mathrm{II}} m_{\mathrm{V}}+J_{\mathrm{II}} m_{\mathrm{II}} m_{\mathrm{VI}}+J_{\mathrm{III}} m_{\mathrm{V}} m_{\mathrm{VI}}$ (where $m_{i}$ is the magnetic moment of $\mathrm{C}_{i}, i=\mathrm{I}, \ldots, \mathrm{VI}$ ). Due to the longer distance between $\mathrm{C}_{\mathrm{III}}$ and $\mathrm{C}_{\mathrm{IV}}\left(\mathrm{C}_{\mathrm{V}}\right.$ and $\left.\mathrm{C}_{\mathrm{VI}}\right)$, we can anticipate that $\left|J_{\mathrm{III}}\right| \ll\left|J_{\mathrm{I}}\right|,\left|J_{\mathrm{II}}\right|$. The $\mathrm{C}_{\mathrm{III}}$ and $\mathrm{C}_{\mathrm{V}}$ (similarly $\mathrm{C}_{\mathrm{IV}}$ and $\mathrm{C}_{\mathrm{VI}}$ ) atoms will always carry magnetic moments in the same direction, which is due to the antipattern rule, and have been verified in previous studies of pristine ZGNRs, which leads $m_{\mathrm{III}}=m_{\mathrm{V}}$ and $m_{\mathrm{IV}}=m_{\mathrm{VI}}$. Due to symmetric consideration, we obtain $\left|m_{\mathrm{I}}\right|=\left|m_{\mathrm{II}}\right|$ and $\left|m_{\mathrm{III}}\right|=\left|m_{\mathrm{IV}}\right| \neq 0$. Therefore, we can examine three different cases as follows.

(1) $m_{\mathrm{I}}=m_{\mathrm{II}}=0: E_{1}=2 J_{\mathrm{III}} m_{\mathrm{III}} m_{\mathrm{IV}}$. Since $J>0$, then we can deduce that $m_{\mathrm{III}}=-m_{\mathrm{IV}}\left(\mathrm{C}_{\mathrm{III}}\right.$ and $\mathrm{C}_{\mathrm{IV}}$ should couple antiferromagnetically with each other). This indicates antiferromagnetic coupling between the two zigzag edges of the ZGNR.

(2) $m_{\mathrm{I}}=m_{\mathrm{II}} \neq 0: E_{2}=J_{\mathrm{I}} m_{\mathrm{I}}^{2}+2 J_{\mathrm{II}} m_{\mathrm{I}}\left(m_{\mathrm{III}}+m_{\mathrm{IV}}\right)+$ $2 J_{\mathrm{III}} m_{\mathrm{III}} m_{\mathrm{IV}}$. The favorable magnetic coupling state is $m_{\mathrm{III}}=m_{\mathrm{IV}}$ and $m_{\mathrm{III}} \times m_{\mathrm{I}}<0$. This will lead to 


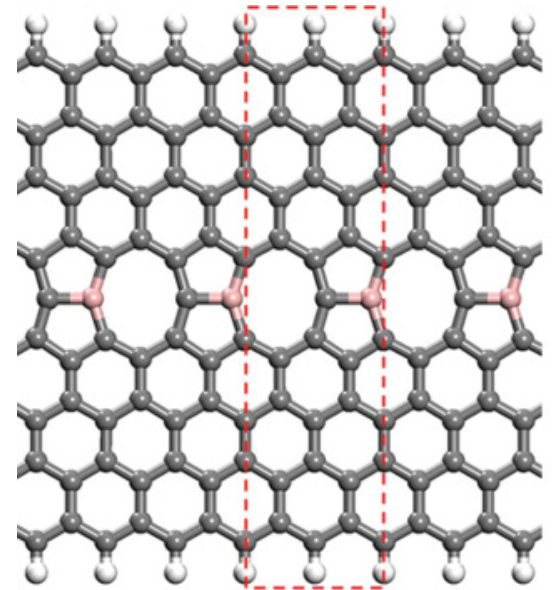

(a)

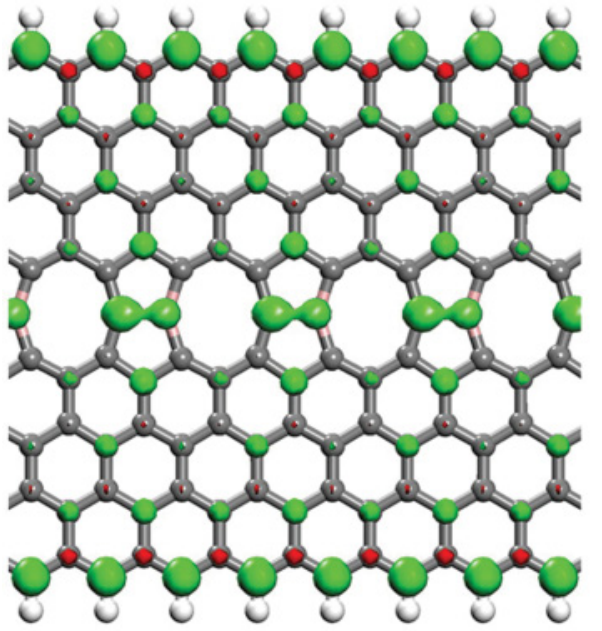

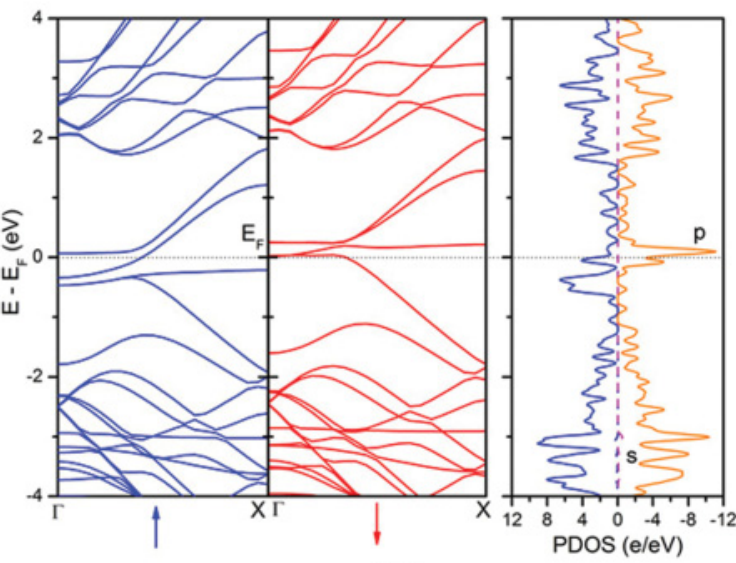

(b)

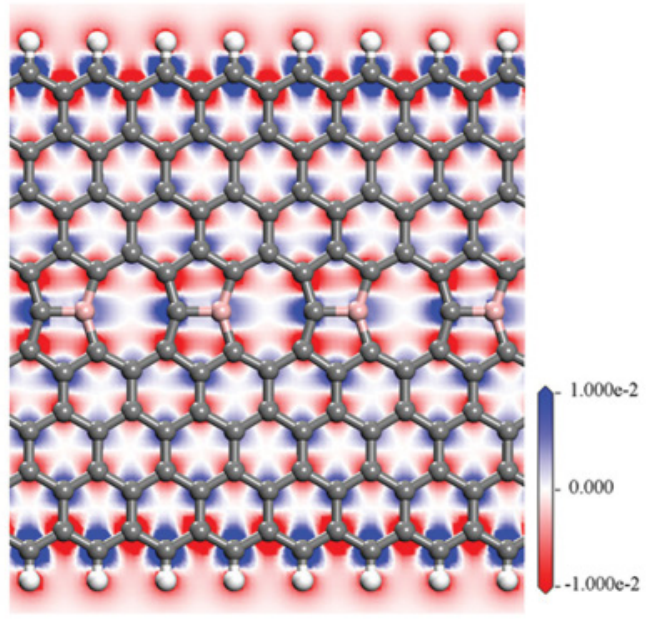

(c)

FIG. 4. (Color online) (a) Geometric structure, (b) band structure and PDOS, and (c) spin density of B-doped 558-defect 9-ZGNR.

ferromagnetic coupling between the two zigzag edges of the ZGNR.

(3) $m_{\mathrm{I}}=-m_{\mathrm{II}} \neq 0: E_{3}=-J_{\mathrm{I}} m_{\mathrm{I}}^{2}+2 J_{\mathrm{III}} m_{\mathrm{III}} m_{\mathrm{IV}}$. Since $\left|J_{\text {III }}\right| \ll\left|J_{\mathrm{I}}\right|$, this case will always possess higher energy than the above two cases.

The relative energy arrangement between case (1) and case (2) is determined by the ratio between exchange parameters. It can be easily deduced that when $\left|J_{\mathrm{II}} / J_{\mathrm{I}}\right|>\left|m_{\mathrm{I}} / 4 m_{\mathrm{III}}\right|$, then $E_{2}<E_{1}$ and vice versa. In its free state the exchange interaction between $\mathrm{C}_{\mathrm{I}}$ and $\mathrm{C}_{\mathrm{II}}\left(J_{\mathrm{I}}\right)$ is large, so that it prefers case (1) instead of case (2). However, when the uniaxial tension is applied, the $J_{I}$ becomes smaller due to longer $\mathrm{C}_{\mathrm{I}}-\mathrm{C}_{\mathrm{II}}$ bond length and weaker interaction between them; then the inequality satisfies, and case (2) is more favorable.

The band structure and PDOS for $c=5.15 \AA$ are given in Fig. 3(b). It shows that the system becomes metallic in both spin-up and spin-down channels, and the metallic bands are contributed mainly by $p$ electrons. Such metallic conductivity is different from the semiconducting feature in its free state. Therefore, uniaxial tension can induce a transition from semiconductor to metal in 558-defect-9ZGNR.

From the previous discussion we can see that uniaxial tension is effective for inducing ferromagnetism. A basic question follows: Is it also possible to induce ferromagnetism in defect-free ZGNR through uniaxial tension? Additional calculations have been performed on $8-Z$ GNR where there are eight zigzag chains, which can be derived from 558-defect$9-$ ZGNR by removing the defects. The calculated results are shown in Fig. 2(b). It is found that the AFM state is always the ground state. Therefore ferromagnetism cannot be induced in pristine defect-free ZGNRs just by applying uniaxial tension. This demonstrates the positive role of the defect line in manipulating the magnetic properties.

\section{Strategy II: Substitutional doping of foreign atoms}

In our previous strategy the transition to FM is achieved by introducing structural distortion of the nanoribbons. Here we study another method to modify the magnetic coupling in defects by substitutional doping of $\mathrm{B}, \mathrm{N}, \mathrm{Al}$, and $\mathrm{P}$ in the $\mathrm{C}_{\mathrm{II}}$ atom, which have different atomic sizes and different electron configurations compared with $\mathrm{C}$. They are selected since their valence electron numbers differ from $\mathrm{C}$ by only one, which may induce magnetism to the defect line. In fact, doping foreign atoms into pristine graphene sheet has been successfully achieved in experiments. ${ }^{32-36}$ 


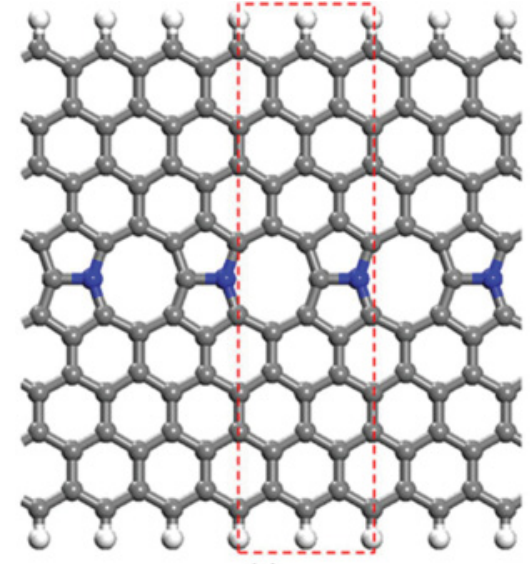

(a)

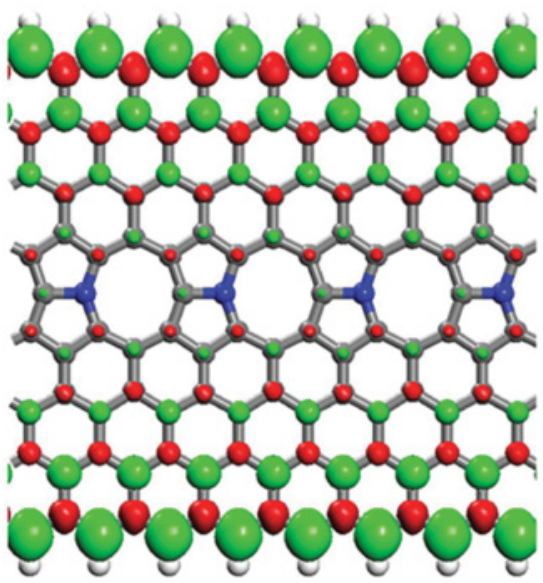

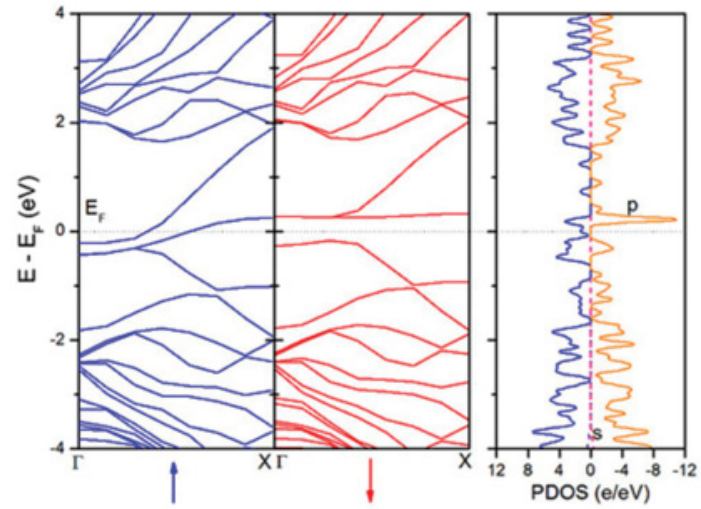

(b)

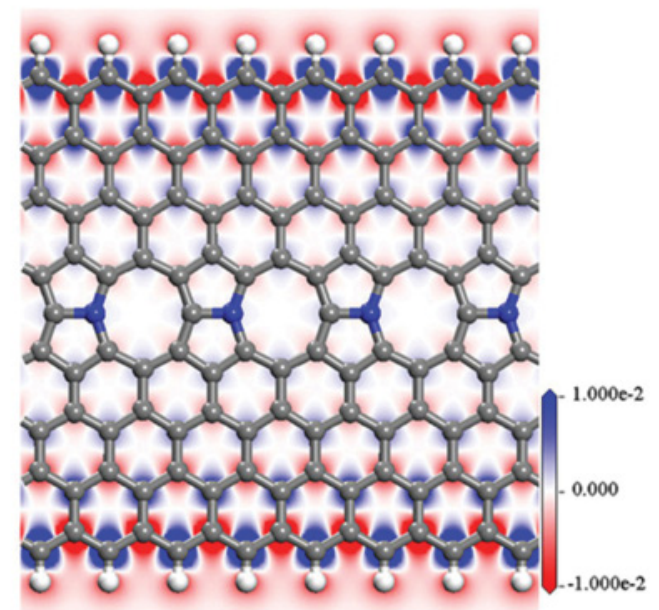

(c)

FIG. 5. (Color online) (a) Geometric structure, (b) band structure and PDOS, and (c) spin density of N-doped 558-defect 9-ZGNR.

When $B$ substitutes the $\mathrm{C}_{\mathrm{II}}$ atom of the 558-defect-9-ZGNR [Fig. 4(a)], we find that the system retains its planar structure, and the $\mathrm{C}_{\mathrm{I}}$-B bond length is $1.555 \AA$, larger than the critical $\mathrm{C}_{\mathrm{I}}-\mathrm{C}_{\mathrm{II}}$ bond length of $1.455 \AA$ at $c^{0}=5.03 \AA$, as shown in Fig. 2(c). This suggests that $B$ doping can also expand the bond length of $\mathrm{C}_{\mathrm{I}}-\mathrm{B}$ defects similar to the uniaxial tension, as discussed in the previous strategy. The $\mathrm{C}_{\mathrm{I}}$ and $\mathrm{B}$ atoms carry magnetic moment of 0.268 and $0.182 \mu_{\mathrm{B}}$, respectively. The exchange energy between the two edges is calculated to be $+21 \mathrm{meV}$, indicating stable FM coupling. Band structure

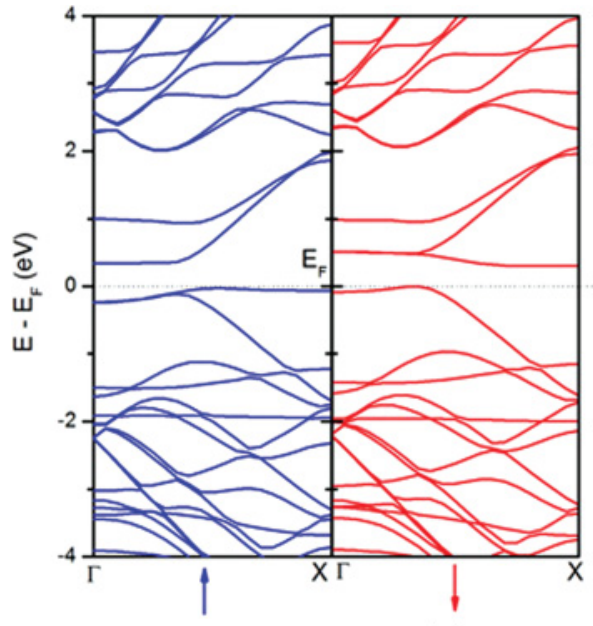

(a)

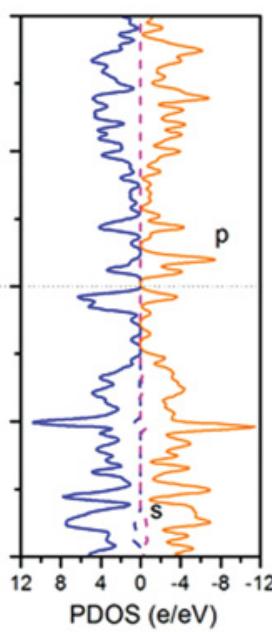

PDOS (e/eV)

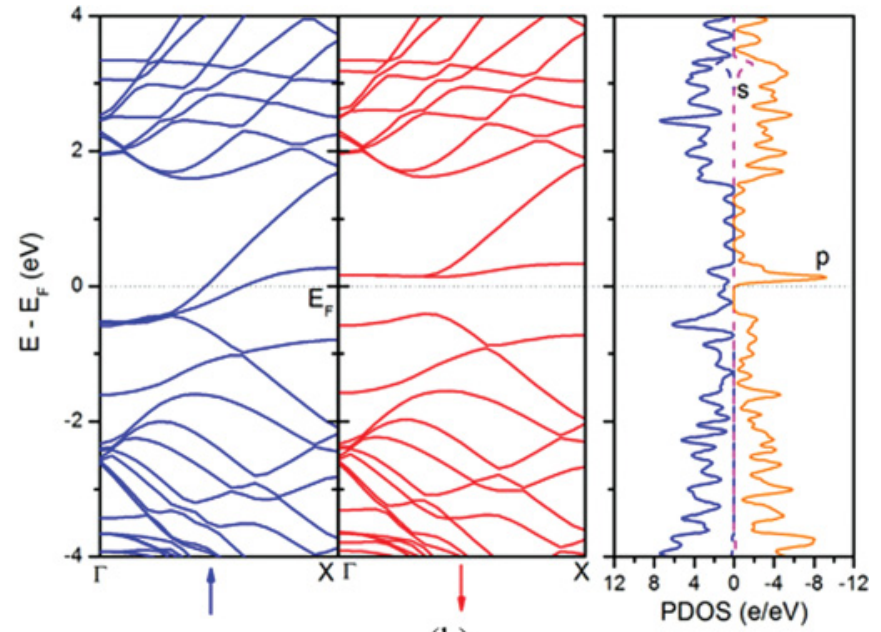

(b)

FIG. 6. (Color online) Band gap and PDOS of (a) Al and (b) P-doped 558-defect 9-ZGNR. 
and PDOS show that the system is metallic in both spin channels, contributed by $p$ electrons [Fig. 4(b)]. From the spin density given in Fig. 4(c), we can see that both $C_{I}$ and $B$ are magnetic and coupled ferromagnetically with each other. The total magnetic moment is calculated to be $1.74 \mu_{\mathrm{B}}$ per unit cell, which is significantly enhanced as compared to the case under uniaxial tension. To investigate the stability of the substituted 558-defect-9-ZGNR, we calculate the formation energy, which is defined as $E_{\mathrm{F}}=\left(E_{\text {substituted }}+E_{\mathrm{C}}\right)-\left(E_{558}+E_{\text {foreign }}\right)$, where $E_{\text {substituted }}$ and $E_{\text {foreign }}$ are the total energy of the doped system and the foreign atom $(\mathrm{B}, \mathrm{N}, \mathrm{Al}$, or $\mathrm{P})$, respectively. Positive formation energy means endothermic reaction process, while negative value corresponds to exothermic reaction. All the formation energies of the four substituted systems are found to be positive, suggesting they are all endothermic. Besides, we have performed molecular dynamics simulation using Nose-Hoover heat bath at $300 \mathrm{~K}$ to check the thermodynamic stability of our systems. We found that all of the systems, including the 558-defect-9-ZGNR and the substituted nanoribbons, are stable with very slight distortions, indicating that they can be applicable under room temperature.

Similarly, for the N-doped 558-defect-9-ZGNR (Fig. 5), we note that since the $\mathrm{N}$ atom has one more $p$ electron than the $\mathrm{C}$ atom, the $\mathrm{C}_{\mathrm{I}}$ and $\mathrm{N}$ atoms are also magnetic with magnetic moment of 0.012 and $0.001 \mu_{\mathrm{B}}$, respectively. The $\mathrm{C}_{\mathrm{I}}-\mathrm{N}$ bond length is $1.425 \AA$, slightly less than the $\mathrm{C}_{\mathrm{I}}-\mathrm{C}_{\mathrm{II}}$ bond length due to the smaller radius of $\mathrm{N}$. The exchange energy in this case is $12 \mathrm{meV}$, which is much less than that in the B-doped case. This is due to the small $\mathrm{C}_{\mathrm{I}}-\mathrm{N}$ bond length and small magnetic moment carried by the $\mathrm{C}_{\mathrm{I}}$ and $\mathrm{N}$ atoms. The total magnetic moment is calculated to be $0.997 \mu_{\mathrm{B}}$, and the system is found to be half metallic with metallic spin-up channel and semiconducting spin-down channel (band gap of $0.42 \mathrm{eV}$ ).

For the Al- or P-doped structure, similar to B or N doping, the two edges of the system also become FM coupled. We find that the Al-doped system is semiconducting with band gap of $0.30 \mathrm{eV}$, while the P-doped system is half metallic (metallic spin-up channel and semiconducting spin-down channel with band gap of $0.54 \mathrm{eV}$ ), similar to $\mathrm{N}$ doping (Fig. 6). The bond length between the $C_{I}$ and the foreign atom, the total magnetic moment, the formation energy, and the exchange energy are summarized in Table I. We can see the following features. (1) When B or Al is substituted, the magnetic moment carried by the defect line is much larger than those of $\mathrm{N}$ - or P-doped

TABLE I. The bond length between the $\mathrm{C}_{\mathrm{I}}$ and the foreign atom $\left(R_{1}\right.$; in $\left.\AA\right)$, the magnetic moment of $\mathrm{C}_{\mathrm{I}}\left(m_{\mathrm{CI}}\right.$; in $\left.\mu_{\mathrm{B}}\right)$ and the doped atom $\left(m_{\mathrm{D} / \mathrm{CII}}\right.$; in $\left.\mu_{\mathrm{B}}\right)$, the total magnetic moment $\left(m_{\text {total }}\right.$; in $\left.\mu_{\mathrm{B}}\right)$, the formation energy $\left(E_{\mathrm{F}}\right.$; in $\mathrm{eV} /$ unit cell), and the exchange energy $(\Delta E$; in $\mathrm{meV})$ per unit cell of 558-defect-9-ZGNR.

\begin{tabular}{lccccc}
\hline \hline & $\mathrm{C}$ & $\mathrm{B}$ & $\mathrm{N}$ & $\mathrm{Al}$ & $\mathrm{P}$ \\
\hline$R_{1}$ & 1.432 & 1.555 & 1.425 & 1.920 & 1.699 \\
$m_{\mathrm{CI}}$ & 0.000 & 0.268 & 0.012 & 0.271 & 0.001 \\
$m_{\mathrm{D} / \mathrm{CII}}$ & 0.000 & 0.182 & 0.001 & 0.015 & -0.001 \\
$m_{\text {total }}$ & 0.000 & 1.737 & 0.997 & 1.000 & 1.000 \\
$E_{\mathrm{F}}$ & - & 1.97 & 2.34 & 7.89 & 5.99 \\
$\Delta E$ & -14 & 21 & 12 & 43 & 6 \\
\hline \hline
\end{tabular}

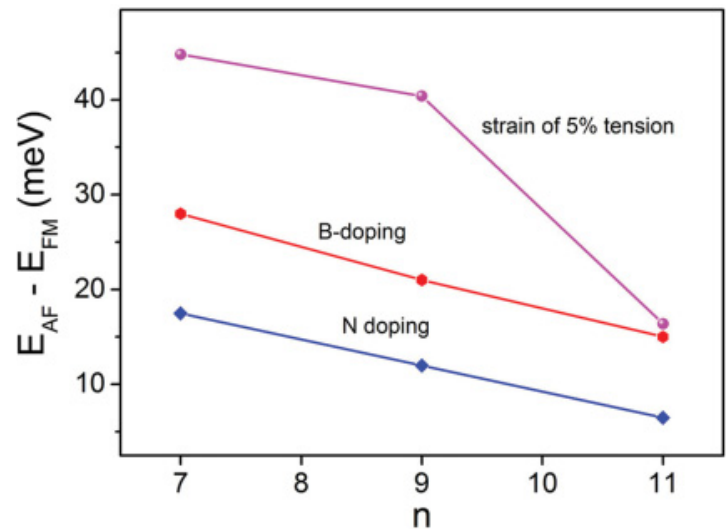

FIG. 7. (Color online) Exchange energy dependence on the width of 558-defect- $n$-ZGNR.

systems. (2) The $\mathrm{C}_{\mathrm{I}}-\mathrm{B}(\mathrm{Al})$ bond is longer than that of the $\mathrm{C}_{\mathrm{I}}-\mathrm{N}(\mathrm{P})$ bond. (3) Hence, the $\mathrm{B}$ and $\mathrm{Al}$ substitution is much more effective than the $\mathrm{N}$ and $\mathrm{P}$ to induce $\mathrm{FM}$ coupled edges. (4) Compared with the defect-free case,${ }^{10}$ the FM stability in ZGNRs with defect lines has been considerably improved by carrier doping due to the different mechanisms.

\section{Dependence of exchange energy on the width of nanoribbon}

In this section we present the exchange energy as a function of the width of the 558-defect- $n$-ZGNR for $n$ going from 7 to 11 for different strategies, as discussed previously. The results are given in Fig. 7. We see that the exchange energies are always positive, indicating that the edges are ferromagnetically coupled. As $n$ gets larger, the exchange energy gradually decreases due to weaker magnetic coupling across the wider nanoribbon. We find the following sequence of the exchange energy for specific widths of the 558-defect $n$-ZGNRs: $\Delta E($ uniaxial strain $)>\Delta E($ B-doping $)>\Delta E(\mathrm{~N}$-doping $)$.

\section{CONCLUSIONS}

We studied the transition from AFM to FM in the edge-coupled configuration of nanoribbons with line defects of 558-type, which has been experimentally fabricated. By considering the magnetic property of $\mathrm{C}_{\mathrm{I}}$ and $\mathrm{C}_{\mathrm{II}}$, we have explored two possible pathways including (i) applying uniaxial tension along the periodic direction and (ii) substituting $\mathrm{C}_{\mathrm{II}}$ with $\mathrm{B}, \mathrm{N}, \mathrm{Al}$, or $\mathrm{P}$ atoms. The underlying mechanism is that the induced magnetism at $\mathrm{C}_{\mathrm{I}}$ ( or $\mathrm{C}_{\mathrm{II}}$ ) mediates the ferromagnetic coupling across the edges. Compared to the existing methods proposed for such magnetic transition, the present strategies have some additional advantages: (1) larger exchange energies for stabilizing ferromagnetic phase and (2) using defects as additional variables to modulate magnetism. In fact, defects exist in many cases. In addition, the Curie temperature can be roughly estimated by mean field theory which states $T_{\mathrm{C}}=$ $\Delta E / k_{\mathrm{B}}$. In this case the Curie temperatures of strained ZGNR (4\%), B, N, Al, and P substituted systems are $464 \mathrm{~K}$, $244 \mathrm{~K}, 139 \mathrm{~K}, 499 \mathrm{~K}$, and $70 \mathrm{~K}$, respectively. Experiments as carried out by $\mathrm{C}$. Tao et al. ${ }^{24}$ (detecting $d I / d V$ spectra of 558-defect-ZGNR) can be used to check our predictions. We 
hope that the present theoretical study would stimulate new experimental efforts in this direction.

\section{ACKNOWLEDGMENTS}

This work is partially supported by the National Natural Science Foundation of China (Grant No. NSFC-21173007), from the National Grand Fundamental Research 973 Program of China (2012CB921404), and from the United States Department of Energy. The authors thank the crew of the Center for Computational Materials Science, the Institute for Materials Research, Tohoku University (Japan), for their continuous support of the HITACHSR11000 supercomputing facility.
*The first two authors contributed equally to this work.

${ }^{\dagger}$ Corresponding author: sunqiang@pku.edu.cn

${ }^{1}$ K. S. Novoselov, A. K. Geim, S. V. Morozov, D. Jiang, Y. Zhang, S. V. Dubonos, I. V. Grigorieva, and A. A. Filrsov, Science 306, 666 (2004).

${ }^{2}$ A. K. Geim, Science 324, 1530 (2009).

${ }^{3}$ L. Tapasztó, G. Dobrik, P. Lambin, and L. P. Biro, Nat. Nanotechnol. 3, 397 (2008).

${ }^{4}$ S. S. Datta, D. R. Strachan, S. M. Khamis, and A. T. CharlieJohnson, Nano Lett. 8, 1912 (2008)

${ }^{5}$ C. Berger, Z. Song, X. Li, X. Wu, N. Brown, C. Naud, D. Mayou, T. Li, J. Hass, A. N. Marchenkov, E. H. Conrad, P. N. First, and W. A. de Heer, Science 312, 1191 (2006).

${ }^{6}$ Y.-W. Son, M. L. Cohen, and S. G. Louie, Phys. Rev. Lett. 97, 216803 (2006).

${ }^{7}$ O. V. Yazyev and M. I. Katsnelson, Phys. Rev. Lett. 100, 047209 (2008).

${ }^{8}$ E. H. Lieb, Phys. Rev. Lett. 62, 1201 (1989).

${ }^{9}$ J. Zhou, Q. Wang, Q. Sun, and P. Jena, Phys. Rev. B 84, 081402(R) (2011).

${ }^{10}$ K. Sawada, F. Ishii, M. Saito, S. Okada, and T. Kawai, Nano Lett. 9, 269 (2009)

${ }^{11}$ K. Sawada, F. Ishii, and M. Saito, Phys. Rev. B 82, 245426 (2010)

${ }^{12}$ M. Wang and C. M. Li, New J. Phys. 12, 083040 (2010).

${ }^{13}$ J.-A. Yan and M. Y. Chou, Phys. Rev. B 82, 125403 (2010).

${ }^{14}$ Z. Li, W. Zhang, Y. Luo, J. Yang, and J. G. Hou, J. Am. Chem. Soc. 131, 6320 (2009).

${ }^{15}$ B. Xu, J. Yin, Y. D. Xia, X. G. Wan, K. Jiang, and Z. G. Liu, Appl. Phys. Lett. 96, 163102 (2010).

${ }^{16}$ X. Lin and J. Ni, Phys. Rev. B 84, 075461 (2011).

${ }^{17}$ J. Lahiri, Y. Lin, P. Bozkurt, I. I. Oleynik, and M. Batzill, Nat. Nanotechnol. 5, 326 (2010).
${ }^{18}$ S. Okada, K. Nakada, K. Kuwabara, K. Daigoku, and T. Kawai, Phys. Rev. B 74, 121412(R) (2006).

${ }^{19}$ L. Kou, C. Tang, W. Guo, and C. Chen, ACS Nano 5, 1012 (2011).

${ }^{20}$ D. Gunlycke and C. T. White, Phys. Rev. Lett. 106, 136806 (2011).

${ }^{21}$ A. R. Botello-Méndez, X. Declerck, M. Terrones, H. Terronesa, and J.-C. Charlier, Nanoscale 3, 2868 (2011).

${ }^{22}$ N. D. Mermin and H. Wagner, Phys. Rev. Lett. 17, 1133 (1966).

${ }^{23}$ O. V. Yazyev, Rep. Prog. Phys. 73, 056501 (2010).

${ }^{24}$ C. Tao, L. Jiao, O. V. Yazyev, Y. Chen, J. Feng, X. Zhang, R. B. Capaz, J. M. Tour, A. Zettl, S. G. Louie, H. Dai, and M. F. Crommie, Nat. Phys. 7, 616 (2011).

${ }^{25}$ C. N. R. Rao, H. S. S. Ramakrishna Matte, K. S. Subrahmanyam, and U. Maitra, Chem. Sci. 3, 45 (2012).

${ }^{26}$ J. P. Perdew, K. Burke, and M. Ernzerhof, Phys. Rev. Lett. 77, 3865 (1996).

${ }^{27}$ B. Delley, J. Chem. Phys. 92, 508 (1990).

${ }^{28}$ B. Delley, J. Chem. Phys. 113, 7756 (2000).

${ }^{29}$ H. J. Monkhorst and J. D. Pack, Phys. Rev. B 13, 5188 (1976).

${ }^{30}$ J. Zhou, K. Lv, Q. Wang, X. S. Chen, Q. Sun, and P. Jena, J. Chem. Phys. 134, 174701 (2011).

${ }^{31}$ T. M. Hattox, J. B. Conklin Jr., J. C. Slatera, and S. B. Trickey, J. Phys. Chem. Solids 34, 1627 (1973).

${ }^{32}$ X. Wang, X. Li, L. Zhang, Y. Yoon, P. K. Weber, H. Wang, J. Guo, and H. Dai, Science 324, 768 (2009).

${ }^{33}$ L. Ci, L. Song, C. Jin, D. Jariwala, D. Wu, Y. Li, A. Srivastava, Z. F. Wang, K. Storr, L. Balicas, F. Liu, and P. M. Ajayan, Nat. Mater. 9, 430 (2010).

${ }^{34}$ H. M. Jeong, J. W. Lee, W. H. Shin, Y. J. Choi, H. J. Shin, J. K. Kang, and J. W. Choi, Nano Lett. 11, 2472 (2011).

${ }^{35}$ B. Guo, Q. Liu, E. Chen, H. Zhu, L. Fang, and J. R. Gong, Nano Lett. 10, 4975 (2010).

${ }^{36}$ X. Wei, M.-S. Wang, Y. Bando, and D. Golberg, ACS Nano 5, 2916 (2011). 